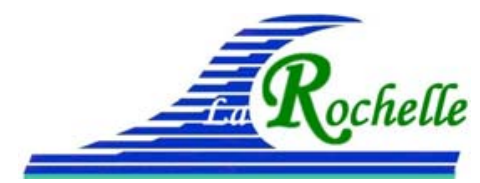

XVèmes Journées Nationales Génie Côtier - Génie Civil

La Rochelle, 29 au 31 mai 2018

DOI:10.5150/jngcgc.2018.085

(C) Editions Paralia CFL

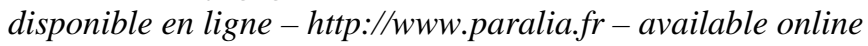

\title{
Comparaison des franchissements et de l'érosion sur le littoral cannois en présence d'ouvrages submergés et de haut de plage (merlons)
}

\section{Florian BREHIN ${ }^{1,2}$, Pierre FARNOLE ${ }^{2}$, Philippe MERCERY ${ }^{1}$, Philippe COESPEL $^{1}$, Dominique BRUN ${ }^{1}$, Gaetan DUFOUR ${ }^{2}$}

1. ICTP, 90 avenue Notre Dame, 06700 Saint-Laurent-du-Var, France. f.brehin@ictp.fr

2. Biobamb sas, 40/54 avenue Sainte-Marguerite, 06200 Nice, France. biobamb.sas@gmail.com

\section{Résumé :}

Des tas de sable (merlons) sont régulièrement mis en place sur le littoral des Alpes Maritimes et du Var à la fin de la saison touristique afin de protéger des tempêtes, les établissements de plage et les infrastructures situés à l'arrière des plages. Ces techniques souples de protection du littoral utilisées contre le phénomène d'érosion et l'aléa de submersion marine des zones urbaines sont temporaires et ont une durabilité et une efficacité qui varient en fonction de la fréquence et de l'intensité des tempêtes. Ces barrières temporaires peuvent également être réalisées en "big bags" attachés entre eux. Cette publication présente les outils de modélisation numérique utilisés pour le dimensionnement et l'optimisation de ces protections temporaires (modèle hydrodynamique SWASH et modèle de transport sédimentaire XBeach) dans le cadre des travaux de ré-ensablement des plages de la Croisette à Cannes en 2017, ainsi qu'une comparaison avec des essais en modèle physique (2D et $3 \mathrm{D})$. Globalement, une bonne cohérence est observée entre les modélisations numériques et physiques. La mise en place de merlons de protection en big bag ou en sable reste efficace pour des tempêtes d'intensité faible à modérée (T1 à T5). Pour des tempêtes plus sévères (T10), la protection est déterminée par l'évolution morphologique de la plage et les caractéristiques granulométriques. Les résultats des essais ont également démontré que la présence d'ouvrages submergés (géotubes) n'avait pas un impact significatif sur la réduction des débits franchissant.

\section{Mots-clés :}

Technique souple de protection, Génie côtier, Aléa de submersion, Erosion, Hydraulique maritime, Morphologie et Sédimentologie littoral.

\section{Introduction}

Face aux problématiques de submersion et d'inondation durant les tempêtes, les solutions de protection des établissements balnéaires en haut de plage incluent la mise en place de structures en batardeaux, de murets en palplanches ou en gabions etc. Ces 


\section{Thème 7 - Risques côtiers}

solutions, bien qu'efficaces, sont généralement couteuses, non réversibles/démontables et demandent une technicité d'installation avancée. De plus, elles réfléchissent les houles et génèrent donc des problèmes d'affouillement à l'amont et/ou en pied des ouvrages. Dans le cas où un stock sableux est disponible, notamment durant des travaux de ré-ensablement, une alternative consiste à mettre en place des protections temporaires de type tas de sable ou merlons (Figure 1). Ces merlons ne sont en effet qu'une réserve de sable en haut de plage pratiquement toujours prélevé sur le profil de plage pour espérer un effet retardant de la submersion. Une variante consiste en la mise en place de "big bags" remplis de sable qui formeront une barrière physique (qui peuvent être recouverts de sable pour maintenir un aspect esthétique naturel).

Les plages de la Croisette s'étendent sur un linéaire de 1,4 km environ de côte sableuse entre les deux ports de Cannes (Vieux port et Port Canto). Lors des tempêtes, l'action de la houle et de la surcote engendre des pertes sédimentaires se traduisant par un abaissement du profil de plage, un recul du trait de côte et dans certains cas une dégradation des infrastructures du haut de plage. De ce fait, la Ville de Cannes a souhaité étudier des solutions innovantes de protection du littoral de cette zone en mettant en place en 2011 une digue sous-marine en géotextile par des fonds de $-3.50 \mathrm{~m}$ NGF. Afin de compléter le dispositif de protection et réduire au maximum l'impact des coups de mer sur les établissements, d'importants travaux de rechargement en sable de carrière $\left(90000 \mathrm{~m}^{3}\right.$ acheminés par voie terrestre et maritime) ont été engagés durant l'automne-hiver 2017-2018 permettant ainsi d'engraisser la plage de façon pérenne. Des merlons ont été installés afin de limiter la submersion en attendant la fin des travaux.
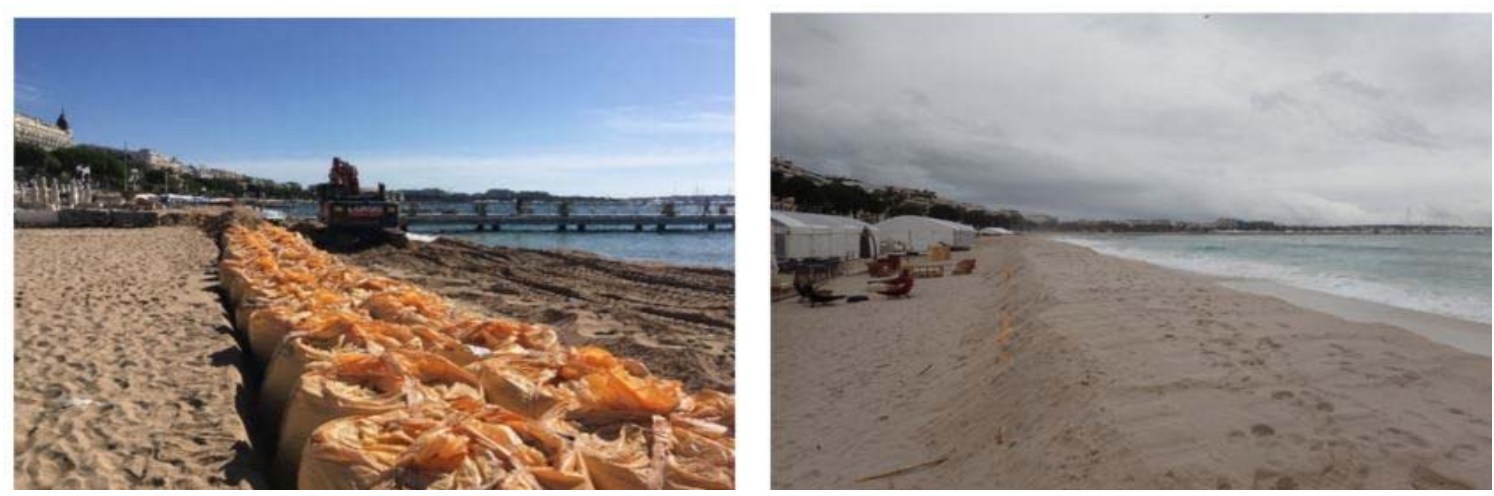

Figure 1. Plages de la Croisette pendant les travaux de ré ensablement et exemple de merlon en big bags mis en place sur le secteur Bâoli (Photographie : ICTP, 2017).

Afin d'assister le maitre d'ouvrage et les gérants d'établissements dans la protection contre l'aléa submersion, des études en modèles physique (canal et bassin) ont été réalisées (OCEANIDE, 2016 ; ANTEA \& ICTP, 2017b). Bien que présentant des avantages certains en terme de reproduction des processus et de compréhension des mécanismes, les essais en modèles réduits présentent des inconvénients (coûts, durée, 


\section{XVèmes Journées Nationales Génie Côtier - Génie Civil \\ La Rochelle, 29 au 31 mai 2018}

limites physiques, contraintes d'échelle, etc.). Les essais physiques peuvent être complétés par des simulations numériques qui permettent de valider les résultats et d'affiner des solutions.

Cet article présente les résultats de modèles numériques 1D (SWASH pour la submersion et XBeach pour l'érosion) mis en œuvre dans le cadre de la maitrise d'œuvre du rechargement des plages de la Croisette. Les simulations ont notamment permis de reproduire les franchissements et d'optimiser le dimensionnement des merlons (les caractéristiques géométriques) ainsi le positionnement le long du profil de plage durant les tempêtes. Les objectifs sont les suivants :

- Quantification de l'aléa submersion (débits franchissant) en fonction des périodes de retour de houle et de niveau d'eau et comparaison avec des résultats de modélisations physiques (canal et bassin);

- Influence des ouvrages submergés (géotubes) et des ouvrages en haut de plage (merlons) sur les franchissements (réduction des débits);

- Quantification de l'aléa érosion en termes d'évolution du profil de plage (transport cross shore) et impact des ouvrages sur la sédimentation

\section{Méthodologie}

\subsection{Description des modèles numériques}

Pour les calculs de submersion, le modèle d'agitation SWASH est implémenté pour la propagation de la houle à la côte. Le code SWASH (Simulating WAves till Shore) est développé par l'université de Delft (Pays Bas) dans la continuité du code SWAN et résout les équations non-linéaires en faible profondeur en y ajoutant un terme de pression non-hydrostatique (ZILEMNA et al., 2011). SWASH permet d'étudier la propagation des vagues, les processus de déferlement et le franchissement des ouvrages côtiers de façon déterministe. Les sorties du modèle incluent les débits franchissant en haut de plage. Le code SWASH a été appliqué auparavant dans des études de propagation de houle pour déterminer le potentiel houlomoteur aux abords d'ouvrages côtiers sur plusieurs sites en France (COIGNARD et al., 2014 ; MICHARD et al., 2016) et dans des études de submersion (LE ROY et al., 2016).

La modélisation de l'érosion est réalisée à l'aide du modèle XBeach, développé conjointement par l'université de Delft (Pays Bas), UNESCO et le corps des ingénieurs de l'armée américaine (USACE). Ce modèle permet de résoudre les équations de propagation des vagues, de transport et de conservation du sédiment (évolution du fond) et est particulièrement adapté pour modéliser l'impact des événements climatiques extrêmes sur les dunes et les plages (ROELVINK et al., 2009). Les modélisations sont réalisées sur une dimension le long de deux profils sélectionnés. Les sorties du modèle incluent les profils de plage avant/après tempête. Cette approche unidimensionnelle a 


\section{Thème 7 - Risques côtiers}

déjà été mise en place pour déterminer l'impact des tempêtes sur le littoral du Pays Basque espagnol (MORICHON et al., 2014).

\subsection{Données d'entrée : profils de plage et conditions hydrodynamiques}

Les simulations numériques ont été réalisées sur un ensemble de profils repartis le long des plages de la Croisette (Figure 2). Cet article se focalise sur les profils 2 et 3 pour représenter respectivement une plage non-protégée et une plage protégée par les géotubes. Les données topo-bathymétriques consistent en une combinaison des données LITTO3D (SHOM) et un levé local de la zone réalisé en 2016.
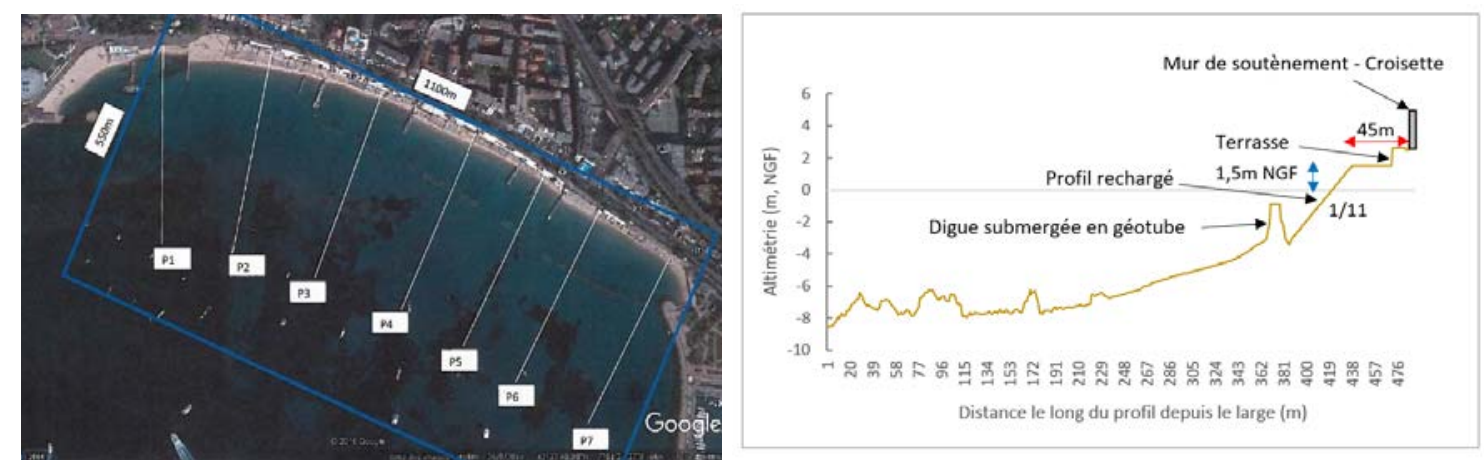

Figure 2. Localisation des profils de plage extraits sur la Croisette (gauche); schématisation du profil de plage P3 pour les modélisations (droite).

Les conditions limites de houle en entrée des modèles SWASH et XBeach ont été déterminées au préalable à l'aide d'une étude statistique et d'un modèle de propagation de houle (ANTEA \& ICTP, 2017a). Les niveaux d'eau extrêmes associés (déterminés statistiquement) sont pris en compte dans les simulations.

\subsection{Configurations testées}

Les configurations testées en modèle numérique incluent :

- Largeurs de plage initiale $(25 \mathrm{~m})$ et rechargée $(45 \mathrm{~m})$

- Plage protégée par géotubes immergés (profil P3) et non protégée (profil P2)

- Plage protégée par un merlon en haut de plage et non protégée (variation de la position d'implantation, géométrie et nature : sable uniquement ou big bags remplis de sable (érosif vs. non érosif)

\subsection{Modélisation physique}

Les essais 3D ont été réalisés dans la cuve à houle de dimensions 30x30m du laboratoire du LPEE (Casablanca, Maroc). Le modèle couvre un linéaire de $5000 \mathrm{~m}$ de la partie centrale des plages de la Croisette, avec une échelle de réduction de 1/60 (ANTEA \& ICTP, 2017b). Il s'agit d'un modèle à fond mobile permettant l'étude des évolutions 


\section{XVèmes Journées Nationales Génie Côtier - Génie Civil \\ La Rochelle, 29 au 31 mai 2018}

morphologiques en fonction des tempêtes. Les fonds ont été reproduits à partir d'un levé topo-bathymétrique réalisé en 2017 dans le cadre de la mission de maitrise d'œuvre. Les fonds sont matérialisés par une chape de ciment lissée jusqu'au haut de plage et incluent les géotubes arasés à $-1 \mathrm{~m}$ NGF. Entre l'isobathe $-7 \mathrm{~m}$ NGF et les ouvrages de bord de plage, la chape est recouverte de sable (matériau mobile).

\section{Résultats}

3.1 Modélisation de la submersion : influence des ouvrages sur les franchissements

L'objectif principal des simulations SWASH est de déterminer les débits franchissant en haut de plage au niveau des terrasses des établissements $(+1,5 \mathrm{~m} \mathrm{NGF})$. Les résultats sont présentés sous forme de graphiques représentant la propagation de la houle le long du profil (en fonction de la profondeur) et l'évolution des débits franchissant (en $\mathrm{m}^{3} / \mathrm{m} / \mathrm{s}$ ) en fonction du temps de simulation (Figure 3 gauche et droite, respectivement).

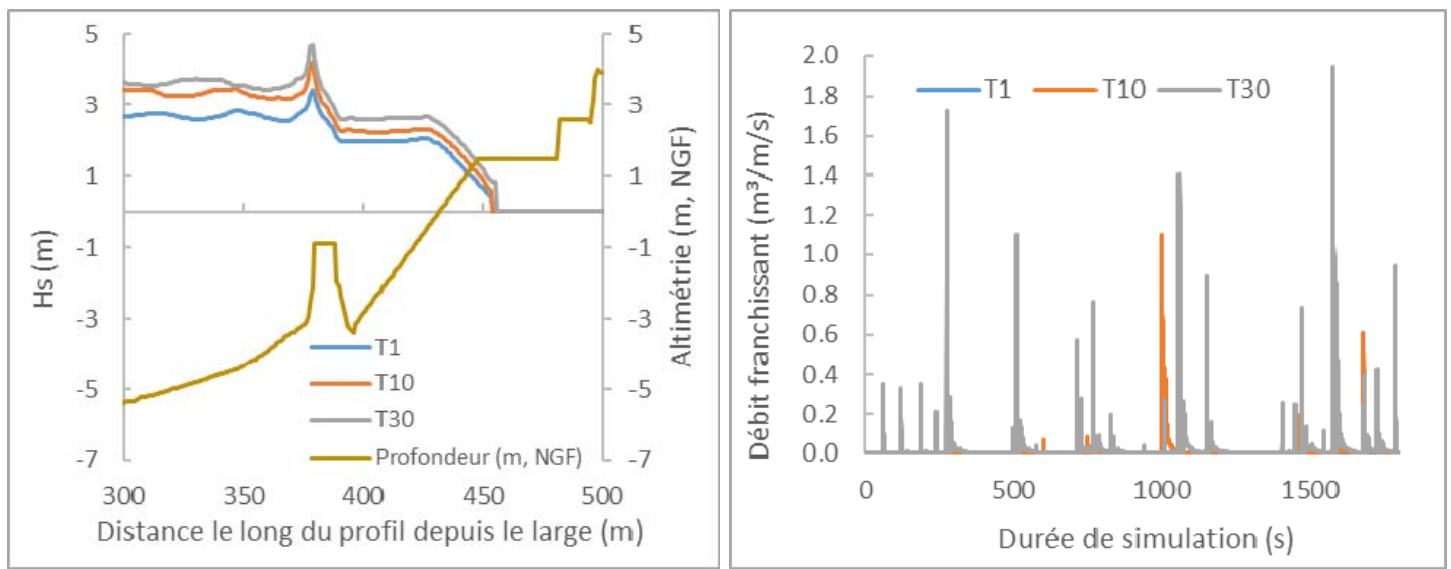

Figure 3. Exemple de résultats de modélisation SWASH. Propagation de la houle le long du profil P3 (gauche); débits franchissant en haut de plage à +1,5m NGF (droite).

SWASH est un modèle non-linéaire qui permet de simuler l'arrivée de trains de houle et l'effet des paquets de mer et les franchissements induits sur une période de temps donnée, similairement à ce qu'il se passe en nature avec des séries de vagues. Les débits franchissant consistent donc en une succession de pics alternant avec des valeurs plus faibles, voire nulles (Figure 3). En effet les franchissements ne se produisent pas forcement pour toutes les vagues, mais seulement durant les séries (groupe de vagues ayant des Hs plus élevées). Les débits moyens calculés par le modèle numérique sur la durée de la simulation sont comparés avec des débits provenant d'essais en canal à houle (OCEANIDE, 2016) réalisés dans le cadre d'un marché antérieur (Tableau 1). Globalement, les valeurs de débits sont en accord et on remarque que l'élargissement de la plage a permis de diminuer significativement les franchissements. 
Tableau 1. Débits franchissant simulés numériquement et en canal à houle.

\begin{tabular}{lrcc}
\hline & & \multicolumn{2}{c}{ Débit moyen $\mathbf{Q}_{\text {moyen }}\left(\mathbf{m}^{3} / \mathbf{m} / \mathbf{s}\right)$} \\
\cline { 3 - 4 } Configuration plage & $\begin{array}{l}\text { Période } \\
\text { de retour } \\
\end{array}$ & $\begin{array}{c}\text { Modèle numérique } \\
\text { (ans) }\end{array}$ & $\begin{array}{c}\text { Canal à houle } \\
\text { (Océanide, 2016) }\end{array}$ \\
\hline Avant rechargement $(25 \mathrm{~m})$ & $T 1$ & 0,191 & 0,311 \\
Avant rechargement $(25 \mathrm{~m})$ & $T 10$ & 1,746 & 2,509 \\
Après rechargement $(45 \mathrm{~m})$ & $T 1$ & 0,011 & 0,039 \\
Après rechargement $(45 \mathrm{~m})$ & $T 10$ & 0,027 & 0,646 \\
\hline
\end{tabular}

L'influence de ouvrages (à la fois submergés et en haut de plage) sur les débits franchissant est illustrée dans le tableau 2. Dans le cas d'une plage protégée par des géotubes (P3), les débits franchissant moyens varient entre $0,011 \mathrm{~m}^{3} / \mathrm{m} / \mathrm{s}$ (T1) et 0,027 $\mathrm{m}^{3} / \mathrm{m} / \mathrm{s}$ (T10). Dans le cas d'une plage non protégée par des géotubes (P2), les débits moyens augmentent de $10 \%$ (T1) à 40\% (T10). Les débits moyens restent du même ordre de grandeur pour une période de retour T30. Dans les 2 cas (protection ou non par géotubes), on note une différence significative entre les débits moyens et maximum pour les conditions sévères T10 et T30 (pratiquement facteur 100) alors que pour T1 les débits sont du même ordre de grandeur. D'autre part, le processus d'atténuation de la houle dû aux géotubes est clairement illustré dans la Figure 3, gauche. Lorsque la plage n'est pas protégée par des géotubes (P2), la houle déferle directement sur la plage sans être amortie, alors que la présence du géotube permet un déferlement en amont de la plage et donc un amortissement des houles.

Tableau 2. Influence des ouvrages submergés (digue géotube) et situés en haut de plage (merlons) sur les débits franchissant à $+1,5 m$ NGF.

\begin{tabular}{|c|c|c|c|c|c|c|c|c|}
\hline \multirow[b]{2}{*}{$\begin{array}{l}\text { Période } \\
\text { retour } \\
\text { (années) }\end{array}$} & \multicolumn{2}{|c|}{$\begin{array}{c}\text { Plage rechargée et } \\
\text { protégée par } \\
\text { géotubes (P3) }\end{array}$} & \multicolumn{2}{|c|}{$\begin{array}{c}\text { Plage rechargée } \\
\text { non protégée par } \\
\text { géotubes }(\mathrm{P} 2)\end{array}$} & \multicolumn{2}{|c|}{$\begin{array}{c}\text { Plage rechargée } \\
\text { protégée par } \\
\text { géotubes }(\mathrm{P} 3)+ \\
\text { merlon }\end{array}$} & \multicolumn{2}{|c|}{$\begin{array}{c}\text { Plage rechargée } \\
\text { non protégée par } \\
\text { géotubes }(P 2)+ \\
\text { merlon }\end{array}$} \\
\hline & $\begin{array}{l}Q \text { moyen } \\
\left(\mathrm{m}^{3} / \mathrm{m} / \mathrm{s}\right)\end{array}$ & $\begin{array}{l}Q \max \\
\left(m^{3} / m / s\right)\end{array}$ & $\begin{array}{l}Q \text { moyen } \\
\left(\mathrm{m}^{3} / \mathrm{m} / \mathrm{s}\right)\end{array}$ & $\begin{array}{l}Q_{\max } \\
\left(m^{3} / \mathrm{m} / \mathrm{s}\right)\end{array}$ & $\begin{array}{l}Q \text { moyen } \\
\left(\mathrm{m}^{3} / \mathrm{m} / \mathrm{s}\right)\end{array}$ & $\begin{array}{l}Q \max \\
\left(m^{3} / \mathrm{m} / \mathrm{s}\right)\end{array}$ & $\begin{array}{l}Q \text { moyen } \\
\left(\mathrm{m}^{3} / \mathrm{m} / \mathrm{s}\right)\end{array}$ & $\begin{array}{l}Q \max \\
\left(m^{3} / m / s\right)\end{array}$ \\
\hline$T 1$ & 0,011 & 0,016 & 0,012 & 0,400 & 0,000 & 0,000 & 0,000 & 0,000 \\
\hline T10 & 0,027 & 1,947 & 0,043 & 1,803 & 0,028 & 1,815 & 0,029 & 1,904 \\
\hline Т30 & 0,029 & 2,203 & 0,055 & 2,005 & 0,033 & 2,247 & 0,058 & 2,122 \\
\hline
\end{tabular}

Concernant l'influence d'un merlon sur les franchissements, dans le cas d'une tempête T1 la houle ne franchit pas le merlon et les débits franchissant n'atteignent pas la terrasse (débits moyens nuls). Par contre, pour des conditions plus sévères (tempêtes T10 et T30), la présence d'un merlon ne permet pas de limiter les débits franchissant, et 


\section{XVèmes Journées Nationales Génie Côtier - Génie Civil \\ La Rochelle, 29 au 31 mai 2018}

au contraire on remarque des débits légèrement plus élevés dus à un écoulement accéléré sur la pente arrière en direction des terrasses. Il faut toutefois noter que les simulations ci-dessus prennent comme hypothèse un merlon non-érosif, et l'érosion et les processus d'affouillement ne sont pas pris en compte. Ces processus sont traités dans le paragraphe suivant.

\subsection{Modélisation de l'aléa érosion : influence des ouvrages sur l'évolution du profil}

L'objectif principal de ces simulations est de déterminer les volumes érodés et l'évolution du profil en fonction des conditions hydrodynamiques. Les simulations permettent également de comprendre l'impact des ouvrages (géotubes et merlons). Les résultats sont présentés sous forme de graphique représentant la profondeur (en m NGF) en fonction de la distance à l'origine du modèle. Chaque graphique inclut le profil initial (profil rechargé avant tempête) matérialisé par une courbe bleue et le profil après tempête représenté par la courbe rouge (Figure 4). Les modélisations 1D ont montré un réel intérêt dans la mise en place de géotubes dans la réduction des pertes volumiques (Tableau 3). Dans le cas d'une période de retour annuelle, les volumes érodés sont de $19,7 \mathrm{~m}^{3} / \mathrm{m}$ le long du profil P2 (non protégée) et de $-12,1 \mathrm{~m}^{3} / \mathrm{m}$ le long du profil P3 protégée par 2 rangées de géotubes. Les tendances de réduction des pertes volumiques sont confirmées pour des conditions plus sévères (T10). Globalement, la mise en place de deux rangées de géotubes entraine un gain en en stabilité d'environ 35\%.

Tableau 3. Volumes érodés le long des profils $P 2$ et P3 en fonction du type de merlon.

Volume érodé au-dessus du contour $0 \mathrm{~m} \mathrm{NGF}\left(\mathrm{m}^{3} / \mathrm{m}\right)$

\begin{tabular}{|c|c|c|c|c|c|c|}
\hline $\begin{array}{l}\text { Période } \\
\text { retour } \\
\text { (années) }\end{array}$ & $\begin{array}{c}\text { Plage } \\
\text { rechargée } \\
\text { (P3) }\end{array}$ & $\begin{array}{c}\text { Plage } \\
\text { rechargée } \\
\text { (P2) }\end{array}$ & $\begin{array}{c}\text { Plage } \\
\text { rechargée } \\
\text { (P3) + } \\
\text { merlon } \\
\text { Big bag }\end{array}$ & $\begin{array}{c}\text { Plage } \\
\text { rechargée } \\
(\mathrm{P} 2)+ \\
\text { merlon } \\
\text { Big bag }\end{array}$ & $\begin{array}{c}\text { Plage } \\
\text { rechargée } \\
\text { (P3) + } \\
\text { merlon } \\
\text { sable }\end{array}$ & $\begin{array}{c}\text { Plage } \\
\text { rechargée } \\
(\mathrm{P} 2)+ \\
\text { merlon } \\
\text { sable }\end{array}$ \\
\hline T1 & $-12,1$ & $-19,7$ & $-12,1$ & $-15,1$ & $-12,1$ & $-16,7$ \\
\hline T10 & $-28,8$ & $-40,0$ & $-15,8$ & $-24,4$ & $-20,9$ & $-32,5$ \\
\hline
\end{tabular}

En ce qui concerne l'influence d'un ouvrage en haut de plage (cas d'un merlon situé à $15 \mathrm{~m}$ de la berme, hauteur de crête à $+2,5 \mathrm{~m}$ NGF et pente de $3 / 2$ ), les volumes érodés le long du profil P3 (plage protégée par des géotubes) sont similaires pour les solutions merlon en sable et merlon en big bag (non-érosif) dans le cas d'une tempête T1. En effet, la vague d'érosion n'a pas encore touché le merlon qui est situé en retrait de l'extrémité de plage (Figure 4, haut). Les différences le long du profil P2 sont visibles dès la période de retour annuelle avec une réduction des pertes volumiques de 25 à $35 \%$. A partir d'une tempête de période de retour T10, le merlon en sable seulement disparait, avec redistribution des sédiments le long du profil (Figure 4, milieu droite). Le merlon 


\section{Thème 7 - Risques côtiers}

en big bags permet de conserver la largeur de plage et surtout de maintenir la hauteur de plage a $+1,5 \mathrm{~m}$ NGF (Figure 4, milieu gauche). L'affouillement au pied d'un merlon en big bag est conséquent au droit de l'ouvrage et pourrait affecter sa stabilité (renversement de big bags).

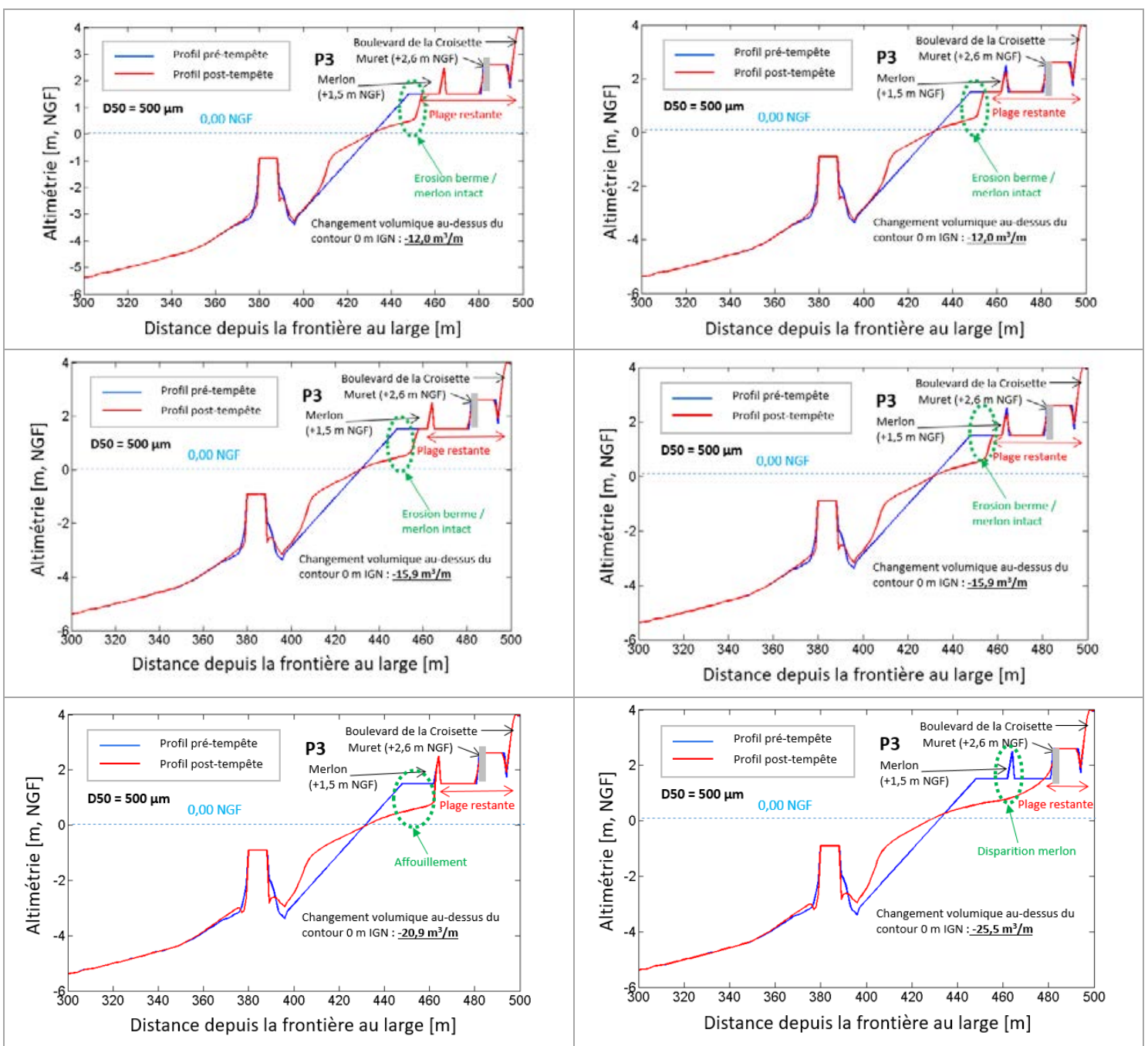

Figure 4. Influence d'un merlon sur les volumes érodés et l'évolution du profil de plage (gauche : merlon en big bag; droite : merlon en sable seulement).

\section{Conclusions et recommandations}

Le dimensionnement $d u$ rechargement de plage et la mise en place d'ouvrages temporaires de protection contre la submersion marine ont été étudiés et optimisés à l'aide de modèles numériques 1D. Les résultats ont montré que les travaux d'élargissement de la plage (largeur de $45 \mathrm{~m}$, à une cote de $+1,5 \mathrm{~m}$ NGF) ont permis de limiter les débits franchissant d'environ $25 \%$ sur le littoral cannois en comparaison avec l'état initial. On note globalement une bonne corrélation entre les débits moyens 


\section{XVèmes Journées Nationales Génie Côtier - Génie Civil \\ La Rochelle, 29 au 31 mai 2018}

calculés par le modèle numérique et les débits observés en canal à houle. Ces débits peuvent atteindre des valeurs considérées comme dangereuses pour les piétons d'après les critères du guide EurOtop (PULLEN et al., 2007) à partir d'une période de retour T10.

Les simulations et observations de terrain ont montré que les débits franchissant sont particulièrement sensibles aux variations de niveau d'eau, en plus des conditions de houle. Par exemple, une tempête de sud début mars 2018 caractérisée par des conditions de houle faibles à modérées $(\mathrm{Hs}=2,4 \mathrm{~m}$ et $\mathrm{Tp}=7 \mathrm{~s}$ ) mais un niveau exceptionnellement haut $(+0,61 \mathrm{~m}$ NGF, correspondant statistiquement à un niveau décennal) dû à la stagnation de basses pressions dans le bassin méditerranéen, a engendré des inondations sur l'ensemble du linéaire des plages de la Croisette ré-ensablées. Les essais en bassin ont montré l'absence de franchissement pour un niveau d'eau de $+0,25 \mathrm{~m}$ NGF alors que la submersion apparait des $+0,5 \mathrm{~m}$ NGF pour une houle annuelle (Figure 5). Les simulations numériques (ANTEA \& ICTP, 2017a) et les essais en bassin (ANTEA \& ICTP, 2017b) ont également montré que la présence d'ouvrages submergés (géotubes) n'avait pas un impact significatif dans la réduction des débits franchissant. Au contraire, une lagune (étendue d'eau) se met en place et stagne en haut de plage provoquant l'inondation des établissements (Figure 5).
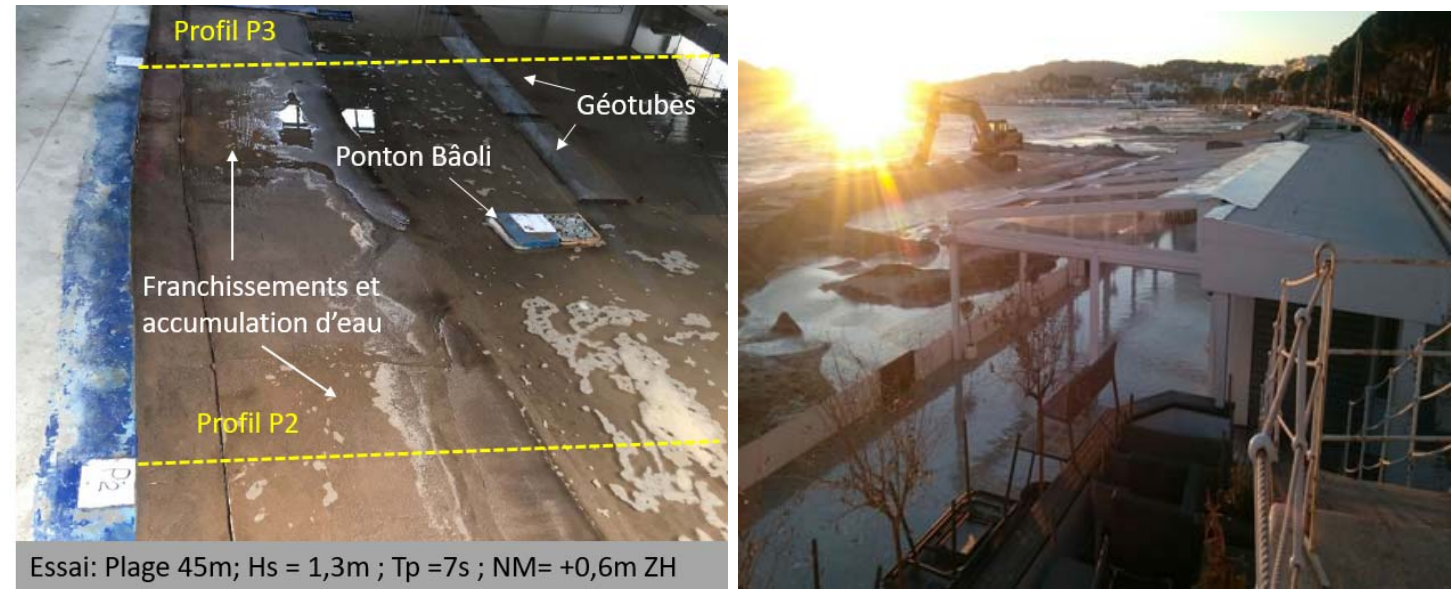

Figure 5. Reproduction en bassin des conditions de franchissement durant la tempête du 03/03/2018 (gauche); photographie prise durant la tempête (droite).

L'article souligne également l'importance de la prise en compte des évolutions morphologiques dans la détermination de l'aléa submersion car la géométrie de la plage et la forme du profil (largeur, pente de plage, etc.), en constante évolution durant les tempêtes, influencent directement le jet de rive et donc les franchissements. Les franchissements sont des processus très complexes et dépendant de nombreux facteurs physiques, qu'il est nécessaire d'appréhender à l'aide d'une combinaison d'essais en modèle physique et numérique. Il est souhaitable d'inclure les évolutions du profil de 


\section{Thème 7 - Risques côtiers}

plage et la géométrie finale dans les calculs de franchissement. La mise en place de merlons de protection en big bag ou en sable reste efficace pour des tempêtes d'intensité faible à modérée ( $\mathrm{T} 1$ à $\mathrm{T}$ ). Pour des tempêtes plus sévères (T10), la protection est déterminée par l'évolution morphologique de la plage et les caractéristiques granulométriques.

\section{Références bibliographiques}

ANTEA \& ICTP (2017a). Etudes préliminaires avec modélisations : Maitrise d'œuvre pour l'aménagement des plages de la Croisette.

ANTEA \& ICTP (2017b). Modélisation bassin 3D: Maitrise d'œuvre pour l'aménagement des plages de la Croisette.

ANTEA \& ICTP (2017c). Rapport d'Avant-Projet: Maitrise d'œuvre pour l'aménagement des plages de la Croisette.

COIGNARD J., MICHARD B., FILIPOT J.F., SERGENT P. (2014). Projet EMACOP : modélisation numérique des vagues à l'approche de la digue d'Esquibien par le code SWASH. XIII ${ }^{\text {èmes }}$ Journées Nationales Génie Côtier - Génie Civil, Dunkerque. pp 45-52 https://doi.org/10.5150/jngcgc.2014.006

LE ROY S., STEPANIAN A., PEDREROS R., BULTEAU T., NICOLAE-LERMA A., BALOUIN Y. (2016). Modélisation numérique à haute résolution de la submersion marine : plaine du Ceinturon, Hyères-les-Palmiers, Var. XIV ${ }^{\text {èmes }}$ Journées Nationales Génie Côtier - Génie Civil, Toulon, pp 689-698. https://doi.org/10.5150/jngcgc.2016.076

MICHARD B., COSQUER E., MALLEGOL A., COIGNARD J., FILIPOT J.F., KPOGO-NUWOKLO K.A., OLAGON M., SERGENT P. (2016). Projet EMACOP : caractérisation des vagues et du potentiel houlomoteur des sites d'Esquibien et de Saint Guénolé par simulation numérique. XIV ${ }^{\text {èmes }}$ Journées Nationales Génie Côtier - Génie Civil, Toulon, pp 497-508. https://doi.org/10.5150/ingcgc.2016.056

MORICHON D., DE SANTIAGO I., ABADIE S. (2014). Modélisation numérique de l'impact des tempêtes sur une plage de poche partiellement aménagée. XIII ${ }^{\text {èmes }}$ Journées Nationales Génie Côtier - Génie Civil, Dunkerque. https://doi.org/10.5150/jngcgc.2014.053 OCEANIDE (2016). Rapport d'essais en canal à houle dans le cadre de la mission complémentaire à la mission d'AMO sur l'aménagement du boulevard de la Croisette.

ROELVINK D., RENIERS A., VAN DONGEREN A., VAN THIEL DE VRIES J., MCCALL R. (2009). Modelling storm impacts on beaches, dunes and barrier islands. Coast. Eng., Vol 56(11-12), pp 1133-1152. https://doi.org/10.1016/j.coastaleng.2009.08.006 PULLEN T., ALLSOP N., BRUCE T., KORTENHAUS A., SCHUTTRUMPF H., VAN DER MEER J.W. (2007). Wave overtopping of sea defences and related structures: assessment manual.

ZIJLEMNA M., STELLING G., SMIT P. (2011). SWASH: An operational public domain code for simulating wave fields and rapidly varied flows in coastal waters. Coast. Eng., Vol. 58, pp 992-1012. https://doi.org/10.1016/i.coastaleng.2011.05.015 